\title{
Early occlusion of the non-infarct-related coronary artery following successful primary percutaneous coronary intervention in ST-elevation myocardial infarction
}

\author{
Rafał Wolny, Jerzy Pręgowski, Paweł Bekta, Zbigniew Chmielak, Adam Witkowski \\ Department of Interventional Cardiology and Angiology, Institute of Cardiology, Warsaw, Poland
}

Postep Kardiol Inter 2015; 11, 2 (40): 136-140 DOI: $10.5114 /$ pwki.2015.52287

\begin{abstract}
A bstract
We present a clinical case of early occlusion of the non-infarct-related artery (non-IRA) in a patient with ST-elevation myocardial infarction (STEMI) treated with primary percutaneous coronary intervention (pPCl). Several hours after successful percutaneous treatment of the occluded right coronary artery the patient developed a second myocardial infarction, which was caused by acute occlusion of the left anterior descending artery, which had a significant lesion in the proximal segment. The lesion was diagnosed during the first catheterization, but was left untreated. We discuss the potential advantages and risks associated with the ad-hoc multivessel PCI strategy in STEMI.
\end{abstract}

Key words: non-infarct-related artery, ST-elevation myocardial infarction, multivessel percutaneous coronary intervention.

\section{Introduction}

The issue of optimal treatment strategy in patients presenting with ST-elevation myocardial infarction (STEMI) and other significant lesions in non-infarct-related arteries (non-IRA) is recently being widely discussed. We hereby present a clinical case of an early occlusion of the non-IRA in a STEMI patient treated with primary percutaneous coronary intervention $(\mathrm{PCI})$.

\section{Case report}

A 50-year-old male heavy smoker with hypertension and hyperlipidemia, without previous chronic medication, was admitted directly to the catheterization laboratory due to recurrent chest pain lasting for 3 days and ST-segment elevation in II, III and aVF ECG leads accompanied by ST-segment depression in I and aVL ECG leads (Figure $1 \mathrm{~A}$ ). $300 \mathrm{mg}$ of acetylsalicylic acid, $600 \mathrm{mg}$ of clopidogrel, 5000 IU of unfractionated heparin and sublingual nitrates were administered in the pre-hospital phase. Due to the preliminary diagnosis of acute myocardial infarction, immediate transfemoral coronary angiography was performed, revealing distal occlusion of the right coronary artery, TIMI 0 (RCA, Figure 1 B) and another significant long lesion in the proximal segment of the left anterior descending artery (LAD) with the first diagonal branch involved, with TIMI 3 flow (Figure $1 \mathrm{D}$, thick arrow). After intravenous administration of eptifibatide (bolus and continuous infusion) the RCA was opened with a guidewire. Pre-dilation with a $2.5 \mathrm{~mm}$ balloon was performed followed by implantation of two drug-eluting stents, $3.0 \times 18 \mathrm{~mm}$ proximally and $2.75 \times 23 \mathrm{~mm}$ distally, with good angiographic effect (Figure $1 \mathrm{C}$ ). The patient felt significant relief of symptoms. The lesion in the LAD was left untreated.

\section{Discussion}

The patient was transferred to the intensive cardiac care unit. Due to blood pressure 180/105 mm Hg and mild residual chest pain he was treated with intravenous nitroglycerin. In echocardiographic examination hypokinetic basal and medial segments of the inferior wall were found, without other abnormalities. After $2 \mathrm{~h}$ the patient had another episode of chest pain, unreactive to increased doses of intravenous nitrates. In ECG new neg-

\section{Corresponding author:}

Rafał Wolny, Department of Interventional Cardiology and Angiology, Institute of Cardiology, 42 Alpejska St, 04-628 Warsaw, Poland, phone: +48 604187 681, e-mail: rafal.wolny@gmail.com

Received: 13.01.2015, accepted: 12.02.2015. 

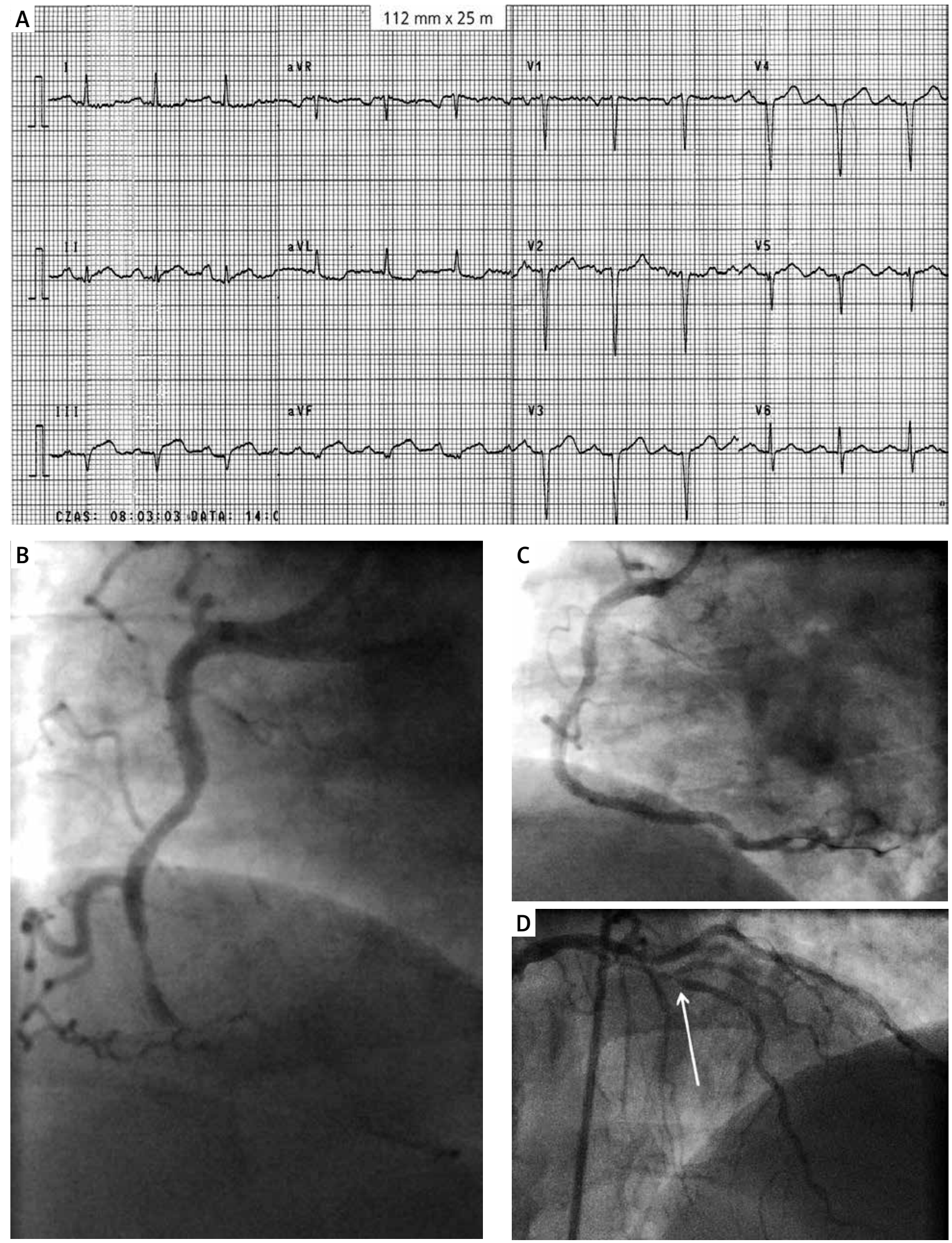

Figure 1. A - ECG at admission showing ST elevation in inferior wall leads. B - Angiography showing occlusion in the mid segment of the RCA; LAO 60, CRAN 0. C - Good result of primary PCI, LAO 60, CRAN 0. D - Angiography showing significant lesion in the proximal LAD; RAO 10, CRAN 30 

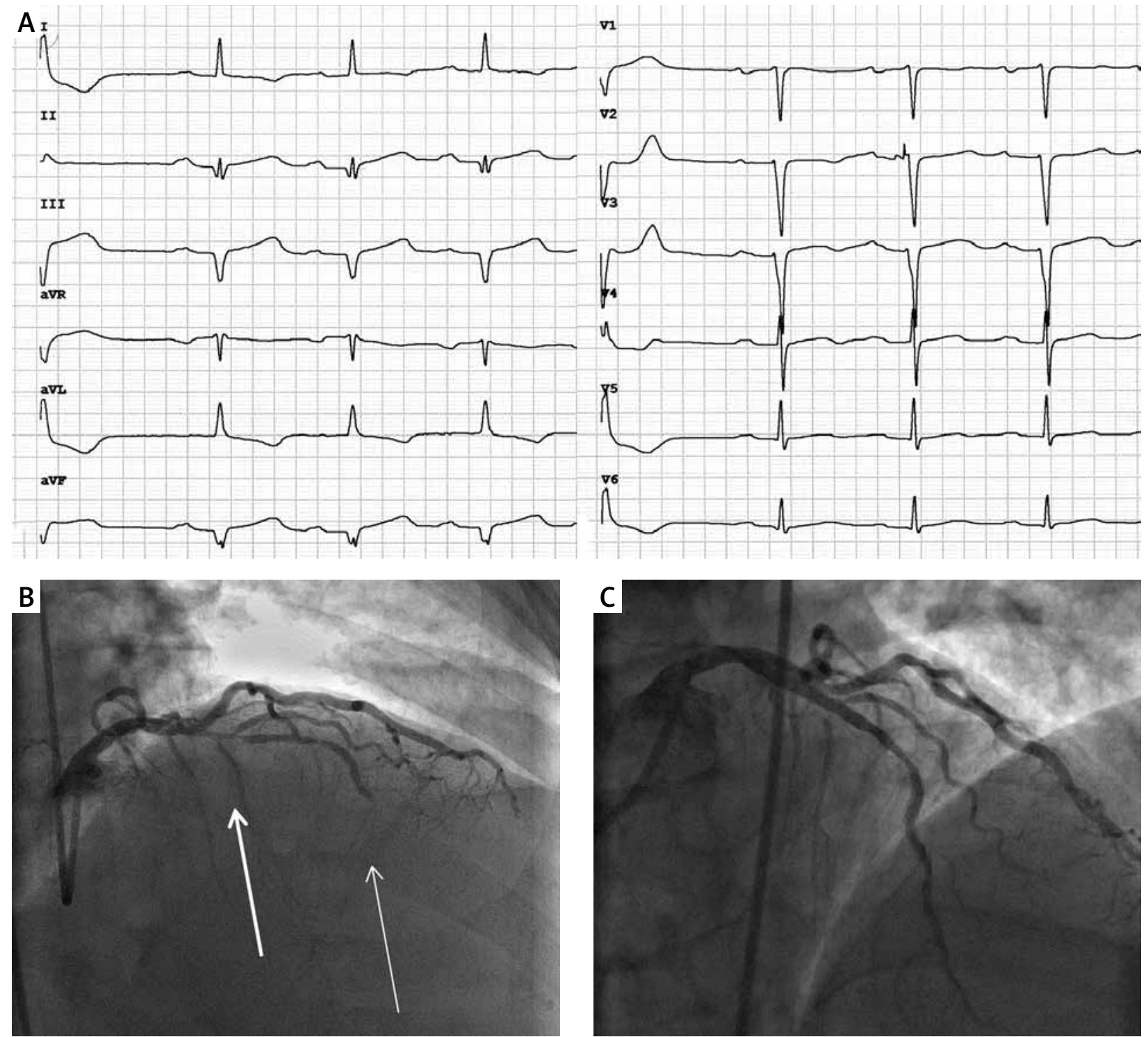

Figure 2. A - ECG after recurrence of angina showing inversion of T waves in antero-lateral leads. B - Angiography showing occlusion in the mid-LAD, RAO 40, CRAN 35. C - Good result of PCI of LAD, CRAN 40

ative or negative/positive T waves in anterior wall leads were found (Figure 2 A). Second coronary angiography was performed showing acute occlusion of the LAD, distally to the significant lesion in the proximal segment (Figure 2 B, significant lesion - thick arrow, occlusion thin arrow). The artery was opened with a guidewire and after pre-dilation with a $2.0 \mathrm{~mm}$ balloon a $2.5 \times 30 \mathrm{~mm}$ drug eluting-stent was implanted in the proximal and medial segment of the LAD, with a good angiographic result (Figure $2 \mathrm{C}$ ). The patient was chest-pain free during ICU observation. Further hospitalization was uneventful, and no new hypokinetic regions of the anterior and lateral wall were found in echocardiography. The patient was discharged home within 6 days.

The issue of selecting the optimal treatment strategy for patients with STEMI and concomitant multiple coronary lesions has been thoroughly discussed to date.
About $40 \%$ of patients with acute $\mathrm{Ml}$ have multivessel disease (MVD) [1]. The prognosis of patients with STEMI and multivessel disease is unfavorable. In the CADILLAC trial the 1-year MACE rate for patients with myocardial infarction and 3-vessel disease was $23.6 \%$ compared with $14.8 \%$ for patients with single-vessel disease [2].

Well-established clinical practice endorsed by European and American guidelines for management of patients presenting with STEMI supports routine $\mathrm{PCl}$ of the culprit lesion only, leaving the other lesions for subsequent evaluation and staged treatment (excluding patients in cardiogenic shock) [3]. This approach is based mainly on the results of data from the HORIZONS-AMI randomized trial, in which patients with MVD and STEMI treated in a single-stage procedure had an unfavorable short- and long-term outcome in comparison to those treated in a staged procedure [4]. Most of the other data regarding 
the clinical outcome of STEMI patients treated in onestage or multi-stage procedures come from non-randomized studies and registries. In the recently published prospective Italian registry REAL that included 2061 patients with STEMI, only 367 patients underwent multivessel $\mathrm{PCl}$ during the index procedure [5]. These patients had significantly higher short-term mortality in comparison to patients who underwent a staged procedure $(\mathrm{HR}=$ $=2.58 ; 95 \% \mathrm{Cl}: 1.06-6.26 ; p=0.03)$. However, there were significant differences between groups, as a single-stage procedure was performed in a generally more diseased population. The comprehensive review and meta-analysis of Bainey et al. [6] that included 46324 patients showed increased in-hospital mortality associated with ad-hoc multivessel PCI (OR = 1.35; 95\% Cl: 1.19-1.54; $p<0.001$ ). However, only 3 of 26 included studies were randomized trials.

Lately, results of the PRAMI trial raised new concerns about the current practice. In this trial 465 patients with STEMI and MVD were randomized $1: 1$ to culprit-only primary $\mathrm{PCl}$ or multivessel $\mathrm{PCl}$ at the index procedure [7]. Patients from the culprit-only group underwent subsequent staged revascularization depending on presence of angina and results of stress testing. So-called preventive multivessel $\mathrm{PCl}$ resulted in significant reduction of the composite end point (cardiac death, non-fatal MI, refractory angina) in comparison with controls ( $H R=0.35$; 95\% Cl: $0.21-0.58 ; p<0.001)$ at a mean follow-up of 23 months, but the study failed to show a significant difference in mortality alone. Also in a large retrospective Korean registry no differences were found in terms of cardiac deaths and adverse cardiovascular events in 1 year between MVD STEMI patients treated in a onestage or a two-stage procedure [8], which may be supportive of performing ad-hoc multivessel $\mathrm{PCl}$. Those new data encouraged authors of the current European guidelines on myocardial revascularization [9] to give recommendation $\mathrm{IlbB}$ to immediate revascularization of significant non-culprit lesions during primary $\mathrm{PCl}$ in selected patients. However, it has not been clearly indicated which patients may benefit from this strategy.

Advisors of one-stage multivessel $\mathrm{PCl}$ argue that it may result in stabilization of disrupted plaques, improvement in left ventricular ejection fraction, and reduction of length of hospital stay and costs of care [10]. They suggest that significant lesions in non-IRA may influence the compensative contractility of the remote myocardial segments. Supporters of the culprit-only strategy point out that the multivessel approach causes administration of a large volume of contrast medium to a usually dehydrated patient and creates a risk of $\mathrm{PCl}$-related complications in the remote myocardial area, including PCI-related myocardial infarction due to occlusion of small side branches. It is also proven that severity of non-culprit lesions in the setting of acute $\mathrm{MI}$ is often overestimated due to vascular constriction [9].

In the present case the patient was treated according to the current STEMI guidelines, and only the IRA underwent stenting during primary $\mathrm{PCl}$, because the flow in the LAD was still TIMI 3 in the presence of a significant lesion. The most usual reason for recurrence of angina symptoms in such a situation would be early stent thrombosis [11]. However, the patient developed early acute occlusion of the non-IRA despite receiving antiplatelets, unfractionated heparin and eptifibatide during $\mathrm{PCl}$. The possible mechanism of LAD occlusion may be distal embolization from unstable atherosclerotic plaque seen in the proximal portion of the vessel. It has been hypothesized that acute myocardial infarction is a state of particularly intense inflammatory response causing instability of coronary plaques in the whole coronary tree. In a recent OCT study by Galon et al. [12], non-culprit coronary plaques in STEMI patients had thinner fibrous caps and contained more macrophages, which made them potentially rupture-prone in comparison to plaques found in stable patients. It is impossible to predict the outcome of the present patient if preventive $\mathrm{PCl}$ of the $\mathrm{LAD}$ lesion was performed during primary $\mathrm{PCI}$. Since the plaque was probably unstable and lipid-rich, wiring, balloon inflations and stenting could have possibly resulted in distal embolization as well. The further clinical course of the patient was good due to the rapid decision of re-angiography and prompt reperfusion after recurrence of ischemic symptoms.

In our opinion, based on the present case, preventive $\mathrm{PCl}$ of the non-IRA in STEMI patients should be carefully considered each time a tight concomitant lesion in a large coronary artery is found. However, there is no good evidence on how to select patients for this therapeutic approach. Many experts agree that treatment of flow-limiting lesions should be recommended, but there is no consensus about lesions that cause no obstruction to blood flow. It is possible that physiological assessment of lesion significance may help make the decision about prompt revascularization. A randomized trial, COMPAREACUTE, to be completed in 2018, designed to compare the results of acute multivessel, FFR-guided PCI with the current staged, FFR-guided procedure, might shed light on this issue (NCT01399736). The ongoing CVLPRIT Trial could give a clue as to whether performing multivessel $\mathrm{PCl}$ at one hospitalization is superior to treatment of the IRA only, leaving other lesions for outpatient evaluation with non-invasive measures [13].

The present case also shows the importance of careful management of a patient with STEMI in the first hours even after successful reperfusion, preferentially in an intensive care unit. Any symptoms of recurrent ischemia should be thoroughly examined considering the general coronary instability of a STEMI patient. 


\section{Conflict of interest}

The authors declare no conflict of interest.

\section{References}

1. Goldstein JA, Demetriou D, Grines CL, et al. Multiple complex coronary plaques in patients with acute myocardial infarction. N Engl J Med 2000; 343: 915-22.

2. Sorajja P, Gersh BJ, Cox DA, et al. Impact of multivessel disease on reperfusion success and clinical outcomes in patients undergoing primary percutaneous coronary intervention for acute myocardial infarction. Eur Heart J 2007; 28: 1709-16.

3. Steg PG, James SK, Atar D, et al. ESC Guidelines for the management of acute myocardial infarction in patients presenting with ST-segment elevation. Eur Heart J 2012; 33: 2569-619.

4. Kornowski R, Mehran R, Dangas G, et al. Prognostic impact of staged versus "one-time" multivessel percutaneous intervention in acute myocardial infarction. Analysis from the HORIZONSAMI (Harmonizing Outcomes With Revascularization and Stents in Acute Myocardial Infarction) trial. J Am Coll Cardiol 2011; 58: 704-11.

5. Manari A, Varani E, Guastaroba P, et al. Long-term outcome in patients with ST segment elevation myocardial infarction and multivessel disease treated with culprit-only, immediate, or staged multivessel percutaneous revascularization strategies: insights from the REAL registry. Catheter Cardiovasc Interv 2014; 84: 912-22.

6. Bainey KR, Mehta SR, Lai T, Welsh RC. Complete vs. culprit-only revascularization for patients with multivessel disease undergoing primary percutaneous coronary intervention for ST-segment elevation myocardial infarction: a systematic review and metaanalysis. Am Heart J 2014; 167: 1-14.

7. Wald DS, Morris JK, Wald NJ, et al. Randomized trial of preventive angioplasty in myocardial infarction. N Engl J Med 2013; 369: 1115-23.

8. Lee HW, Hong TJ, Yang MJ, et al. Comparison of infarct-related artery vs multivessel revascularization in ST-segment elevation myocardial infarction with multivessel disease: analysis from Korea Acute Myocardial Infarction Registry. Cardiology J 2012; 19: 256-66.

9. Windecker S, Kolh P, Alfonso F, et al. 2014 ESC/EACTS Guidelines on myocardial revascularization: The Task Force on Myocardial Revascularization of the European Society of Cardiology (ESC) and the European Association for Cardio-Thoracic Surgery (EACTS) Developed with the special contribution of the European Association of Percutaneous Cardiovascular Interventions (EAPCI). Eur Heart J 2014; 35: 2541-619.

10. Alfonso F. Multivessel intervention during primary angioplasty. Too Greedy? JACC Cardiovascular Interventions 2010; 3: 32-4.

11. Tyczynski P, Karcz MA, Kalinczuk L, et al. Early stent thrombosis. Aetiology, treatment, and prognosis. Postep Kardiol Inter 2014; 10: 221-5.

12. Galon MZ, Wang Z, Bezerra HG, et al. Differences determined by optical coherence tomography volumetric analysis in non-culprit lesion morphology and inflammation in ST-segment elevation myocardial infarction and stable angina pectoris patients. Catheter Cardiovasc Interv 2015; 85: E108-15; doi: 10.1002/ ccd.25660.

13. Kelly DJ, McCann GP, Blackman D, et al. Complete Versus culprit-Lesion only PRimary PCI Trial (CVLPRIT): a multicentre trial testing management strategies when multivessel disease is de- tected at the time of primary $\mathrm{PCI}$ : rationale and design. Eurointervention 2013; 8: 1190-8. 